\title{
Structural elucidation of galactomannan from seeds of Crotalaria mucronata Desv. by atomic force microscopy
}

\author{
YONG-LI YANG ${ }^{1}$, SHOU-JUN GUO ${ }^{1}$, ZHENG-XIA ZHANG $^{1}$, \\ YONG ZHANG ${ }^{2}$, ANG LIU ${ }^{2}$, LI-LING XIE ${ }^{2}$ and YU-ZHONG ZHENG ${ }^{1}$ \\ ${ }^{1}$ School of Food Engineering and Biotechnology, Hanshan Normal University, Chaozhou, Guangdong 521041; \\ ${ }^{2}$ Department of Biology, Shantou University, Shantou, Guangdong 515063, P.R. China
}

Received March 30, 2017; Accepted August 23, 2017

DOI: $10.3892 / \mathrm{mmr} .2017 .8280$

\begin{abstract}
A non-ionic water-soluble galactomannan, isolated from the seed endosperm of Crotalaria mucronata Desv., may be an ideal thickener to increase food viscosity. The present study hypothesized that the viscous property of Crotalaria galactomannan may be associated with its spatial structure. Therefore, the structure of Crotalaria galactomannan was elucidated using an atomic force microscope. The results of the present study demonstrated that the polysaccharide consisted of a D-mannose backbone with D-galactose branches, and the D-mannose/D-galactose ratio was 2.375:1. In the three-dimensional structure of Crotalaria galactomannan, the helix was a common secondary structure, containing numerous ring structures of different sizes. In addition, multiple helixes may link together via hydrogen bonding and van der Waals forces, forming aggregations with small rings or spiral windings. The results of the present study indicated that the multiple-branching construction of Crotalaria galactomannan may underlie its viscosity-enhancing properties in the water phase.
\end{abstract}

\section{Introduction}

Galactomannans, being polysaccharides built up of a $\beta$-(1-4)-D-mannan backbone with single D-galactose branches linked $\alpha-(1-6)$, are present in the endosperm of numerous plants, particularly the Leguminosae (1). The ratios of mannose/galactose may differ according to the species (2). Due to their different physicochemical properties, galactomannans

Correspondence to: Dr Yu-Zhong Zheng, School of Food Engineering and Biotechnology, Hanshan Normal University, Science Building, 15 Dongshan Street, Chaozhou, Guangdong 521041, P.R. China

E-mail: zhengyuzhong@gmail.com

Abbreviations: AFM, atomic force microscope; GC, gas chromatography

Key words: Crotalaria mucronata Desv., galactomannan, molecular structure, AFM are frequently used in the pharmaceutical, biomedical, cosmetics and food industries, including as thickeners, gels, flocculating agents and stabilizing agents $(3,4)$. In addition, the bioactivities of galactomannan have been demonstrated, including anticoagulation, immune regulation, anti-ulcer, antiviral, blood sugar-lowering and antioxidant activities (5-10).

For commercial purposes, galactomannans are extracted primarily from Cyamopsis tetragonolobus L. (guar beans), Ceratonia siliqua (locust beans) and Caesalpinea spinosa (tara). However, the sources of these plants are limited, and research has focused on the identification of alternative sources (11). Crotalaria mucronata Desv. is a common and productive herb in the south of China. The seed endosperm of $C$. mucronata has abundant galactomannan, which may be used as a potential thickening agent (12). In the present study, it was hypothesized that the viscous property of Crotalaria galactomannan was associated with to spatial structure. Therefore, it was necessary to elucidate the structure of Crotalaria galactomannan.

Unlike the electron microscope, which provides a two-dimensional projection of sample, the atomic force microscope (AFM) is able to provide a three-dimensional surface profile. Therefore, AFM is widely used in the spatial formation analysis of different polymer chains, including DNA, proteins, polysaccharides and other macromolecular chains (13-15). In the present study, galactomannan isolated from $C$. mucronata was analyzed by gas chromatography (GC) and AFM in order to elucidate the association between the structure and molecular properties of Crotalaria galactomannan.

\section{Materials and methods}

Materials. Mature seeds of C.mucronata were purchased from the Traditional Chinese Medicine Market of Puning (Puning, China). The authentication of plant materials was performed morphologically by Dr Shou-Jun Guo (Hanshan Normal University, Chaozhou, China). The samples were deposited in Department of Biology, Hanshan Normal University.

Isolation and purification of galactomannans. The seeds (20 g) were immersed in $100 \mathrm{ml}$ boiling water for $20 \mathrm{~min}$, and subsequently incubated for $24 \mathrm{~h}$ at room temperature $\left(28^{\circ} \mathrm{C}\right)$. When the seeds had doubled in size, the endosperm was 
manually collected following removal of the peel and embryo. The endosperm was ground and homogenized for $24 \mathrm{~h}$ in $100 \mathrm{ml}$ water at $75^{\circ} \mathrm{C}$. The viscous solution was centrifuged with $3,200 \mathrm{x}$ for $30 \mathrm{~min}$ at $4^{\circ} \mathrm{C}$ and the supernatant was precipitated with $2 \mathrm{X}(\mathrm{v} / \mathrm{v}) 95 \%$ ethanol. The precipitate was collected on a glass filter (fine grade), and washed successively with $75 \%$ ethanol. Finally, the polysaccharide precipitate was dried using a vacuum lyophilizer and weighed.

Conditions for GC. GC experiments were performed on a Shimadzu GC-14A gas chromatograph (Shimadzu Corporation, Kyoto, Japan) equipped with a DB-5MS column (30 $\mathrm{m} \times 0.25 \mathrm{~mm} \times 0.25 \mu \mathrm{m}$ ) and a flame ionization detector, using a high purity of nitrogen as the carrier gas at a flow rate of $8 \mathrm{~cm}^{3} / \mathrm{min}$. The temperature was heated from 180 to $240^{\circ} \mathrm{C}$ at $10^{\circ} \mathrm{C} / \mathrm{min}$, and kept at $240^{\circ} \mathrm{C}$ for $25 \mathrm{~min}$. The vaporizer temperature was $260^{\circ} \mathrm{C}$ and the detector temperature was $240^{\circ} \mathrm{C}$. The detector voltage was $350 \mathrm{~V}$. An aliquot of $15 \mu \mathrm{l}$ was injected for each run. The quantitative analysis of D-mannose and D-galactose by GC was optimized in a concentration range of $0.03-1.00 \mathrm{mg} / \mathrm{ml}$. The calibration curve of D-mannose was $\mathrm{Y}=0.3458 \mathrm{X}+0.1341\left(r^{2}=0.9921\right)$ and that of D-galactose was $\mathrm{Y}=0.9541 \mathrm{X}-0.6234\left(r^{2}=0.9934\right)$.

Sample preparation for AFM. The purified Crotalaria galactomannan was dissolved in deionized water at $90^{\circ} \mathrm{C}$ for $1 \mathrm{~h}$ using a magnetic stirrer, and subsequently cooled to room temperature. The concentrations of galactomannan solution were prepared at 1 and $2 \mu \mathrm{g} / \mathrm{ml}$. A peeled-off mica slide was pretreated with $5 \mu 110 \mathrm{mM} \mathrm{NiNO} 3$ for $1 \mathrm{~min}$, followed by the addition of 1 or $2 \mu \mathrm{g} / \mathrm{ml}$ galactomannan solution on the $\mathrm{Ni}^{+}$-treated mica surface, which was air-dried at room temperature for $10 \mathrm{~min}$. Finally, the mica slide was rinsed with deionized water and dried, and was observed and imaged using the AFM in tapping mode.

Conditions for AFM. The AFM instrument used was a Nanoscope III, a multimode scanning probe microscope equipped with a type EV scanner (Digital Instruments; Bruker Corporation, Billerica, MA, USA). The tapping mode was employed by using etched silicon cantilever probes of $224 \mu \mathrm{m}$ nominal length, at a spring constant of 20-70 N/m and a contact force of 3-4 $\mathrm{nN}$. Images were obtained at ambient temperature and humidity. For any given image, the height was analyzed in at least three distinct regions of the structure using Nanoscope software (version 5.12; Bruker Corporation). Section analysis of AFM allows depth, height, width and angular measurements to be taken.

\section{Results}

Morphology and structure of Crotalaria galactomannan molecular chains. The GC profile of standard oligosaccharides was revealed, including rhamnose, ribose, arabinose, xylose, inositol, D-mannose, D-galactose and glucose (Fig. 1A).

Retention times indicated that galactomannan was composed of D-mannose and D-galactose, and inositol served as an internal control. The chirality of the sugars was confirmed from these standard oligosaccharides (including D-mannose and D-galactose) (Fig. 1B).
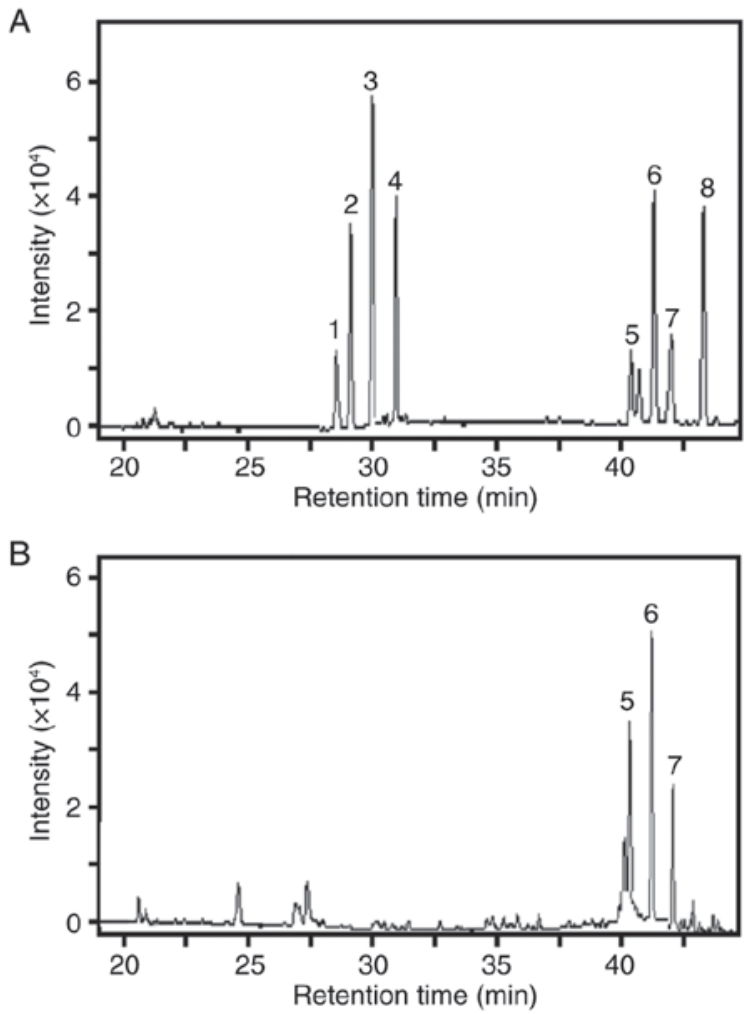

Figure 1. GC analysis of galactomannan from the seed endosperm of Crotalaria mucronata. (A) The GC profile of the standard oligosaccharides was revealed, including rhamnose (1), ribose (2), arabinose (3), xylose (4), inositol (5), D-mannose (6), D-galactose (7) and glucose (8). (B) The GC profile of Crotalaria galactomannan was revealed. According to the retention times, the outstanding peaks were D-mannose (6) and D-galactose (7). Inositol (5) served as an internal control. GC, gas chromatography.

In the GC analysis, the calibration curves of D-mannose and D-galactose were linear over a concentration range of $0.03-1.00 \mathrm{mg} / \mathrm{ml}$. The GC method was precise, accurate and sensitive enough for simultaneous quantitative evaluation of mannose/galactose detection. Peak areas $(n=3)$ of mannose/galactose were used to calculate their contents, and the results indicated that the ratio of D-mannose to D-galactose was $2.375: 1$ in galactomannan (Fig. 1B).

In order to reveal its molecular morphology, $1 \mu \mathrm{g} / \mathrm{ml}$ galactomannan was analyzed by AFM. The two-dimensional and three-dimensional images (Fig. 2) demonstrated that the large molecular chains of polysaccharides exhibited different structures containing numerous rings and branches. The density of the molecular chains depended on the initial concentration of polysaccharide and its deposition amount on the mica chip. The contrast of the image depended on the force on the probe tip of the AFM. Therefore, the optimal force for obtaining a clear and stable three-dimensional image of the polysaccharide was $\sim 3-4 \mathrm{nN}$.

The single chains and multi-branches were observed (Fig. 2A and B). These single polysaccharide chains were able to intertwine to form polymerized molecules. In these polymers, there were numerous different rings that were created at different connections (Fig. 2A and B). In addition, coral-like branches were observed (Fig. 2C). These results indicated that Crotalaria galactomannan may be a single chain containing ring and branch structures. The structure of the single-chain 

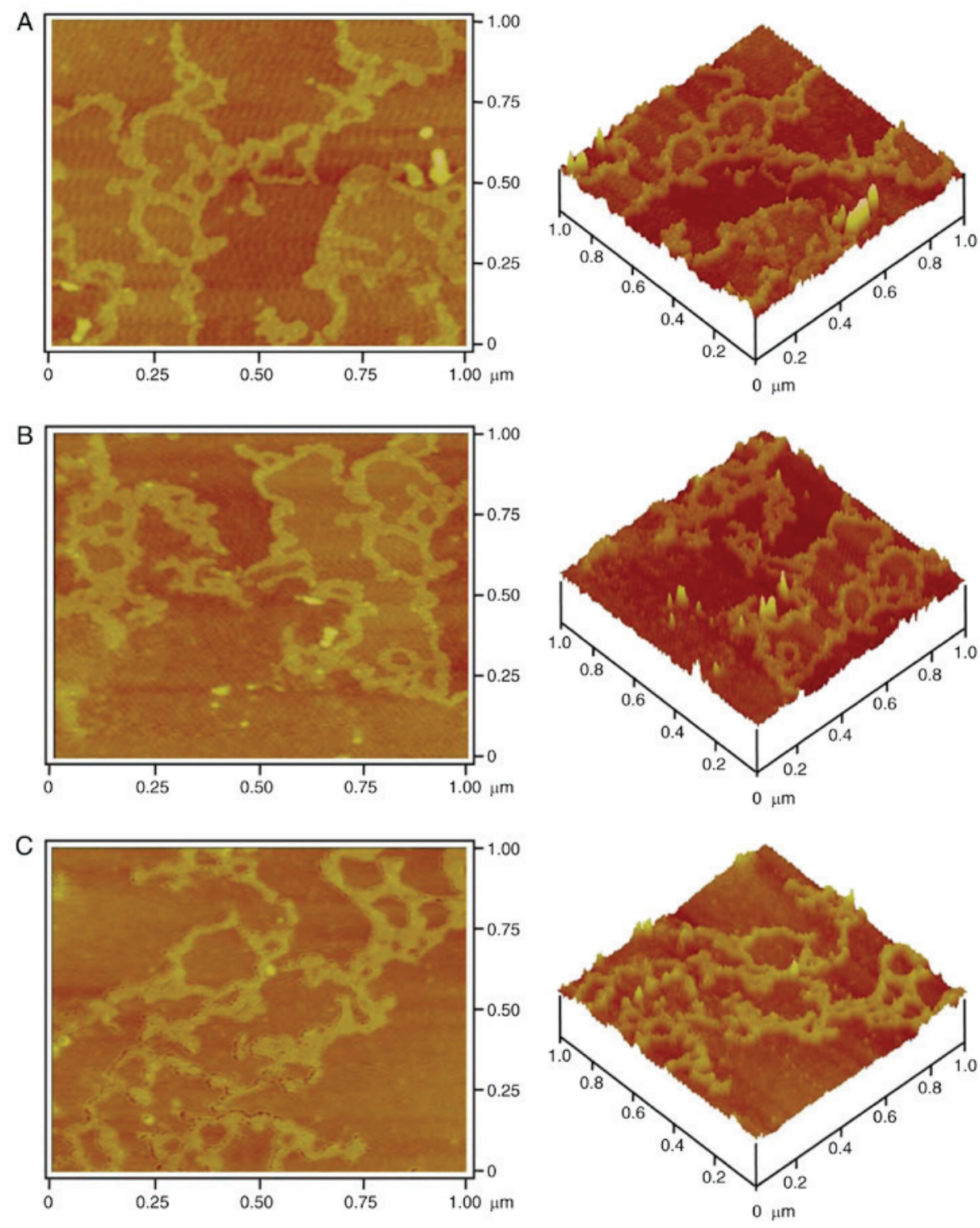

Figure 2. AFM images of Crotalaria galactomannan molecules. (A) Images were captured using AFM of sample I of $1 \mu \mathrm{g} / \mathrm{ml}$ Crotalaria galactomannan. (B) Images were captured using AFM of sample II of $1 \mu \mathrm{g} / \mathrm{ml}$ Crotalaria galactomannan. (C) Images were captured using AFM of sample III of $1 \mu \mathrm{g} / \mathrm{ml}$ Crotalaria galactomannan. The images are presented in one-dimensional (left) and three-dimensional formats (right). AFM, atomic force microscope.

molecule may be affected by interactive forces and the environment; therefore, the appearance of the polysaccharide molecule chain may be changeable. Additionally, polysaccharides may entangle with each other to form the coil structure, finally forming a ring and a short rod-like branch.

Single-molecule structure of Crotalaria galactomannan. Via software analysis, Crotalaria galactomannan was observed to form a single chain ring (Fig. 3A) of 37.34-nm surface width, 37.14-nm horizontal width and 0.10-nm thickness.

Galactomannan was able to form a short single-chain with rod-like branches (Fig. 3B); the surface width of the rod-like branch was $21.62 \mathrm{~nm}$, the horizontal width of the branch was $21.48 \mathrm{~nm}$ and the thickness of the branch was $0.27 \mathrm{~nm}$.

Crotalaria galactomannan may constitute a ring structure with a 101.98-nm diameter (Fig. 3C). The single chains exhibited different properties: On the left side, the surface width was $31.50 \mathrm{~nm}$, the horizontal width was $31.25 \mathrm{~nm}$ and the thickness was $0.10 \mathrm{~nm}$; on the other side, the surface width was $25.80 \mathrm{~nm}$, the horizontal width was $25.39 \mathrm{~nm}$ and the thickness was $0.11 \mathrm{~nm}$.

The software analysis indicated that Crotalaria galactomannan single-chain rod-like branches had a surface width of $27.85 \mathrm{~nm}$, a horizontal width of $27.34 \mathrm{~nm}$ and a thickness of $0.54 \mathrm{~nm}$ (Fig. 3D). Galactomannan was additionally able to form a single chain ring with a diameter of $168.76 \mathrm{~nm}$ (Fig. 3E), and a single chain ring with a surface width of $33.61 \mathrm{~nm}$, a horizontal width of $33.20 \mathrm{~nm}$ and a thickness of $0.26 \mathrm{~nm}$; in addition, there was another single chain with a surface width of $27.29 \mathrm{~nm}$, a horizontal width of $25.39 \mathrm{~nm}$ and a thickness of $0.09 \mathrm{~nm}$.

Spiral-coil structure of Crotalaria galactomannan. A high-resolution image of Crotalaria galactomannan is presented in Fig. 4A. The molecular chain winding and 
A

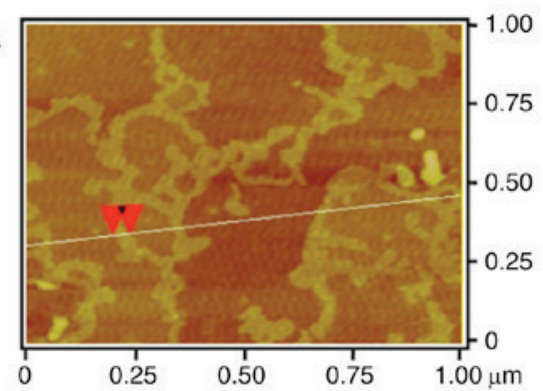

B

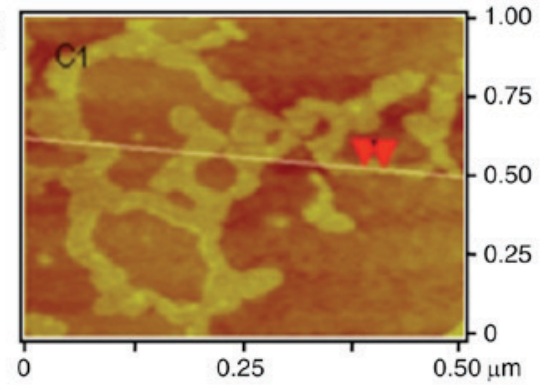

C

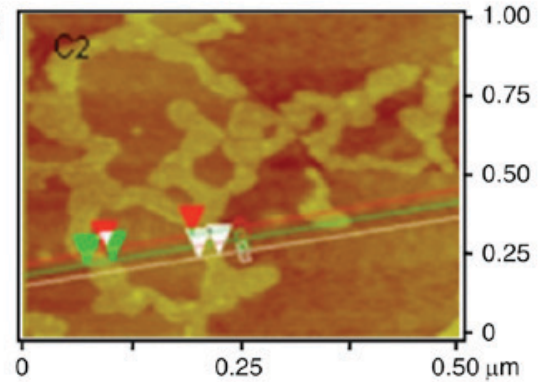

D

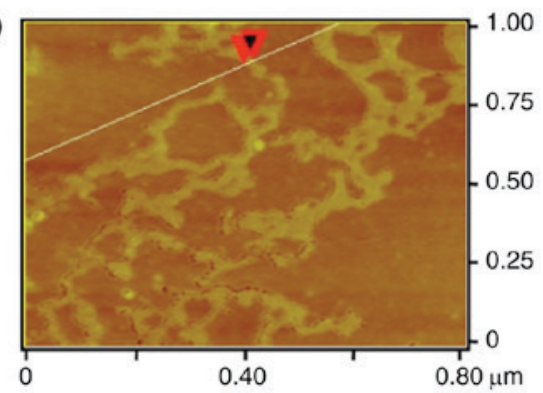

E

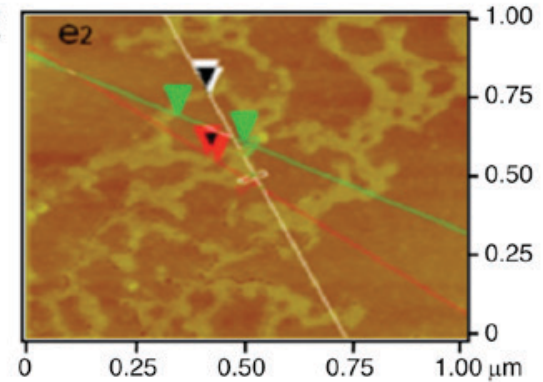

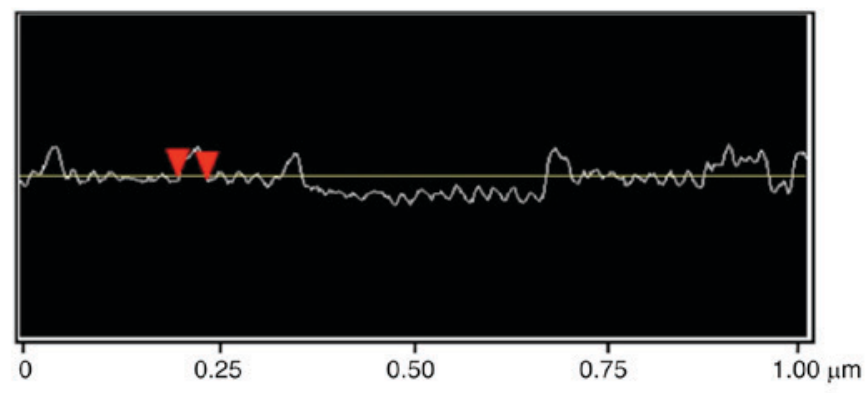
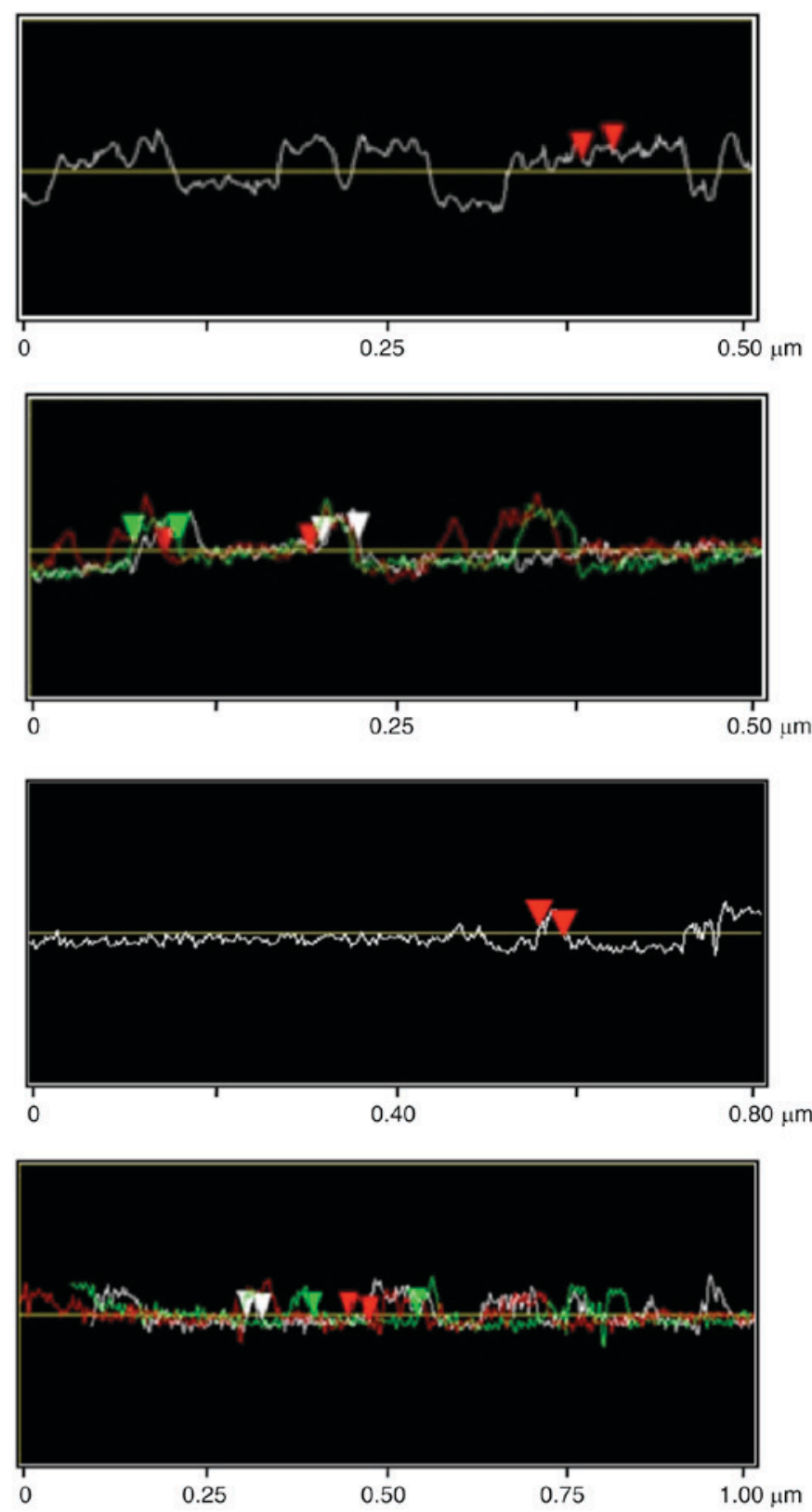

Figure 3. AFM images of Crotalaria galactomannan molecules. Samples of $1 \mu \mathrm{g} / \mathrm{ml}$ Crotalaria galactomannan was photographed by AFM and the images are presented in one-dimensional and three-dimensional formats. The single-molecular structure was analyzed by section analysis. (A) Red arrows indicate a chain thickness of $0.10 \mathrm{~nm}$. (B) Red arrows indicate a rod-like branch thickness of $0.27 \mathrm{~nm}$. (C) Red arrows indicate a ring diameter of $101.98 \mathrm{~nm}$; green arrows indicate a left-chain thickness of $0.10 \mathrm{~nm}$; white arrows indicate a right-chain thickness of $0.11 \mathrm{~nm}$. (D) Red arrows indicate a rod-like chain thickness of $0.54 \mathrm{~nm}$. (E) Green arrows indicate a ring structure diameter of $168.76 \mathrm{~nm}$; red arrows indicate a down-chain thickness of $0.26 \mathrm{~nm}$; white arrows indicate an up-chain thickness of $0.09 \mathrm{~nm}$. AFM, atomic force microscopy.

spiral-coil structure was clearly observed. The surface width of the coil was $22.86 \mathrm{~nm}$, the horizontal width was $22.46 \mathrm{~nm}$ and the thickness was $0.16 \mathrm{~nm}$ (Fig. 4B).
The high-resolution image (Fig. 4B) demonstrated that the single-stranded molecule of the polysaccharide formed an entangled ring and the intramolecular ring of the spiral-coil 
A
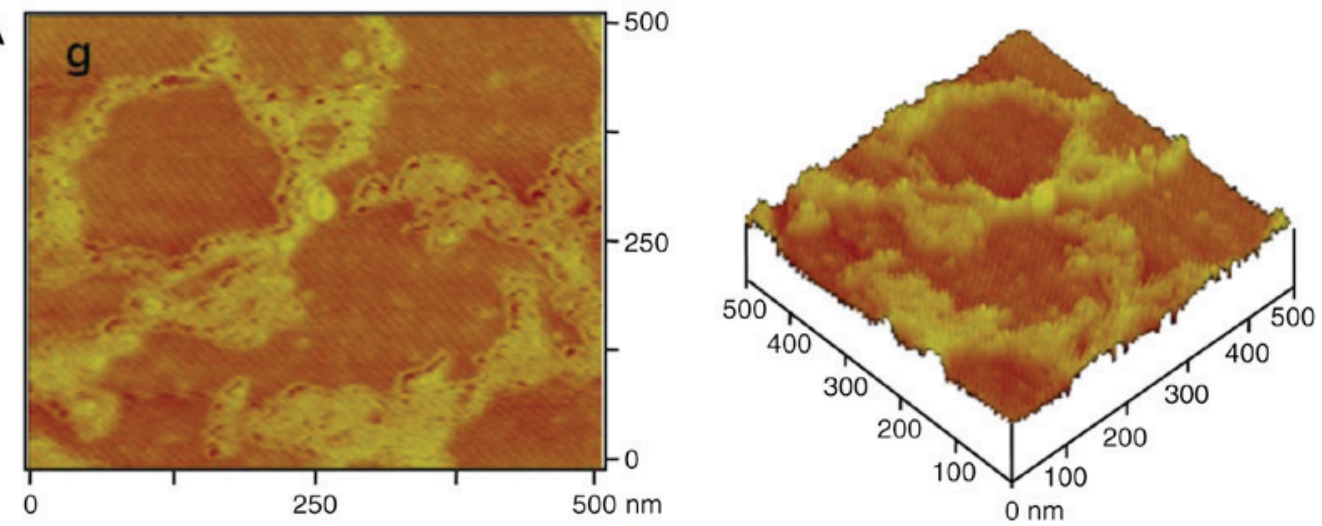

$\mathrm{B}$
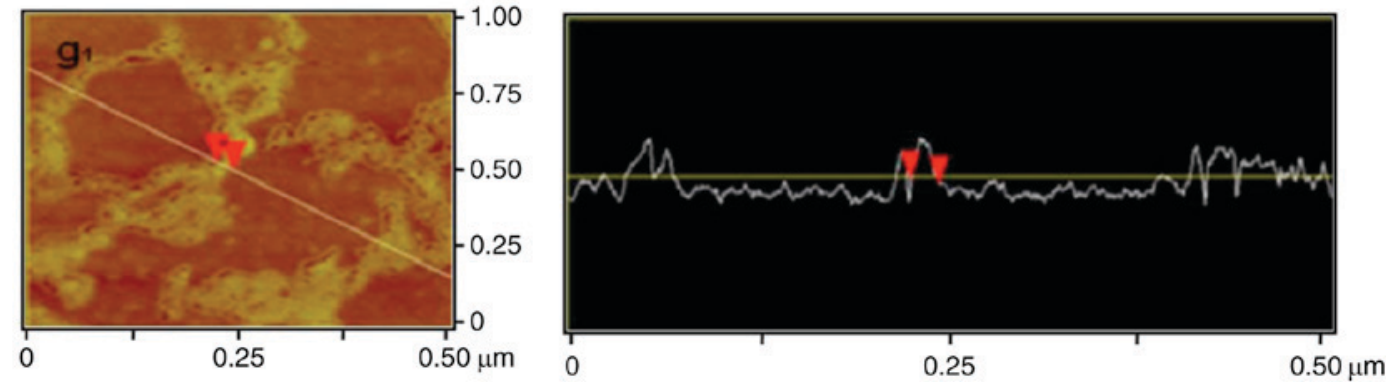

Figure 4. AFM images of Crotalaria galactomannan molecules. (A) High-resolution image of Crotalaria galactomannan captured by AFM. The images are presented in one-dimensional and three-dimensional formats. (B) The single-molecular structure of Crotalaria galactomannan was analyzed by section analysis. The red arrows indicate a chain thickness of $0.16 \mathrm{~nm}$. AFM, atomic force microscope.
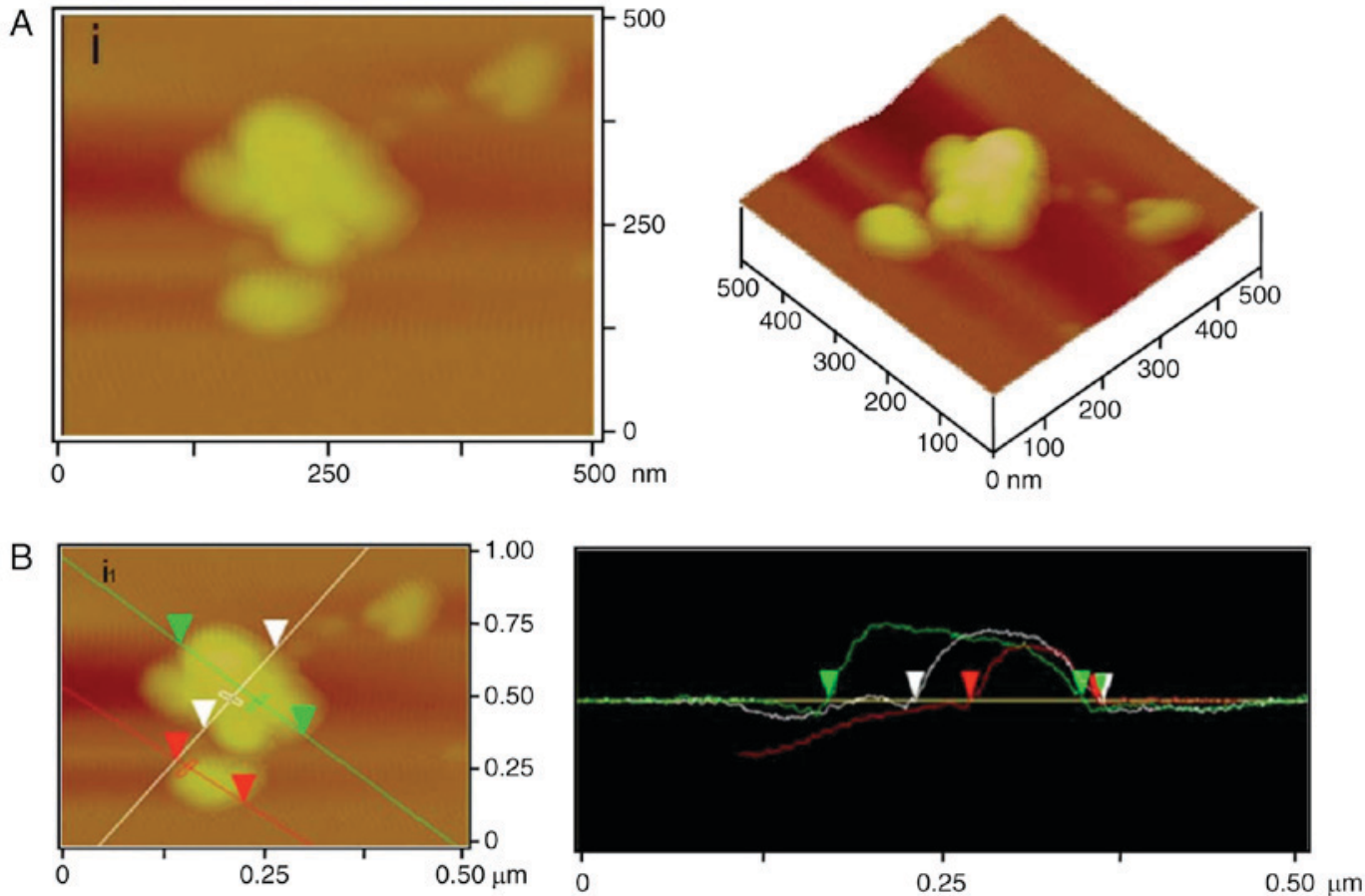

Figure 5. AFM images of macromolecules aggregation of Crotalaria galactomannan. (A) A concentration of $2 \mu \mathrm{g} / \mathrm{ml}$ Crotalaria galactomannan was photographed by AFM. The images are presented in one-dimensional and three-dimensional formats. (B) The multiple-molecular structure of $C r o t a l a r i a$ galactomannan was analyzed via section analysis. Red arrows indicate a spherical aggregation diameter of $110.45 \mathrm{~nm}$; green arrows indicate a spherical aggregation length of $216.10 \mathrm{~nm}$; white arrows indicate a spherical aggregation width of $161.79 \mathrm{~nm}$. AFM, atomic force microscope.

structure. Through the high resolution and software analysis, polysaccharide single-chains were observed form a ring structure of molecules with a winding diameter of $\sim 50-170 \mathrm{~nm}$ and a single-chain length of 1.2-1.5 $\mu \mathrm{m}$ (Fig. 4B).
Macromolecular-aggregation structure of Crotalaria galactomannan. In order to reveal the molecular aggregation of Crotalaria galactomannan, $2 \mu \mathrm{g} / \mathrm{ml}$ galactomannan was analyzed by AFM, producing two-dimensional and 
three-dimensional images (Fig. 5). The results demonstrated that the different-sized polysaccharide macromolecules were able to wind and form spherical aggregates. There were two spherical aggregations which were observed in the images in Fig. 5A. The diameter of the small aggregation was $110.45 \mathrm{~nm}$ and its thickness was $0.086 \mathrm{~nm}$, while the length of large aggregation was $216.10 \mathrm{~nm}$, its width was $161.79 \mathrm{~nm}$ and the thickness was $0.12 \mathrm{~nm}$. The average thickness of the spherical aggregations was $0.10 \mathrm{~nm}$ (Fig. 5B).

This aggregation unit of polysaccharide may be maintained by hydrogen bonds and van der Waals forces, which were the principal components of the spherical structure. In this case, two single-molecule chains may interact with each other to form the double helix of polysaccharide. Additionally, the larger spherical aggregations, higher-level structures, may be composed of four or more globular units of polysaccharide with hydrogen bonds and van der Waals forces.

\section{Discussion}

The three-dimensional structures of polysaccharide are considered to be one of the determinants of their bioactivities, and it has been hypothesized that the high-level structure of a polysaccharide may be more important than the primary structure $(16,17)$. Therefore, it is of importance to study the spatial association between single-chain polysaccharide fragments and their conjugates. The conformational aspects of a polysaccharide include the conformation of each monosaccharide, the orientation of the monosaccharides with respect to each other and the flexibility of the spatial structure, which is defined by dihedral angles, torsion angles around glycosidic bonds, inter-atomic distances and dynamic parameters (17). For Crotalaria galactomannan, the secondary- and higher-level structures in solution are not readily defined, due to their inherent flexibility. The characterization of polysaccharide dynamics by experimental and theoretical means remains an area of active research. With the development of high resolution instrumental processes, including various light scattering techniques (light scattering, X-ray and neutron scattering), X-ray diffraction analysis, small-angle neutron scattering, AFM and high-resolution nuclear magnetic resonance spectroscopy, it may be possible to study the conformation and three-dimensional structure of a polysaccharide at the molecular level (17). The present study used AFM to simulate and visualize the three-dimensional structure of Crotalaria galactomannan.

In AFM observations of Crotalaria galactomannan, the measured values were increased compared with the estimated values for the polysaccharide molecules, which was due to the broadening effect that occurred from scanning different parts of the polysaccharide and the molecular chain. AFM observations of galactomannan may illustrate the structure of a single molecular chain and its various side-chain branches. In addition, the molecular chains may entangle with each other via different interactions, leading to numerous sizes of ring structure, intramolecular spiral structure and spiral-coil structure. The results of the present study thereby demonstrated that the polysaccharide Crotalaria galactomannan exhibited numerous branches.
Multi-strand branches of polysaccharides are tightly arranged and mutually cross-linked, and this type of structure is maintained by intermolecular van der Waals forces and hydrogen bonds (17). Micelle formation results from the effects of the intramolecular force and the presence of micelles adjacent to the polysaccharide chain are conducive to the formation of polysaccharides via hydrogen bonding. A large amount of rod-like polysaccharide scattering was observed on the mica surface in the present study. This rod-like polysaccharide exhibited different lengths, and interacted to form colloidal network structures. The spherical aggregates of polysaccharide may be linked by hydrogen bonding and van der Waals forces. Hydrogen bonds and van der Waals forces could drive two single-molecule chains to associate with a double-helical coil and to form an aggregate. A total of four aggregates with a double-stranded helix globular structure may in turn form a large spherical aggregate of polysaccharides.

In conclusion, the results of the present study demonstrated that Crotalaria galactomannan was composed of one single D-mannose chain and a number of D-galactose branches, and that the D-mannose/D-galactose ratio was 2.375:1. This chain was able to intra-molecularly entangle itself into a helix containing numerous ring structures of different sizes. A group of galactomannan polysaccharides maybe linked together via hydrogen bonding and van der Waals forces to form aggregations with small rings or spiral windings.

\section{Acknowledgements}

The present study was supported by National Natural Science Foundation of China (grant no. 81202907) and the National Natural Science Foundation of Guangdong Province (grant nos. 2012B050300025 and 2014A020221058).

\section{References}

1. Stephen AM, Phillips GO and Williams PA (eds): Food Polysaccharides and Their Applications. Marcel Dekker, Inc., New York, pp155-186, 1995.

2. Kök MS, Hill SE and Mitchell JR: Viscosity of galactomannans during high temperature processing: Influence of degradation and solubilisation. Food Hydrocolloid 13: 535-542, 1999.

3. Srivastava M and Kapoor VP: Seed galactomannans: An overview. Chem Biodivers 2: 295-317, 2005.

4. Vieira ÍGP, Mendes FNP, Gallão MI and de Brito ES: NMR study of galactomannans from the seeds of mesquite tree [Prosopis juliflora (Sw) DC]. Food Chem 101: 70-73, 2007.

5. Hussein MM, Helmy WA and Salem HM: Biological activities of some galactomannans and their sulfated derivatives. Phytochemistry 48: 479-484, 1998.

6. Zia T, Hasnain SN and Hasan SK: Evaluation of the oral hypoglycaemic effect of Trigonella foenum-graecum L. (methi) in normal mice. J Ethnopharmacol 75: 191-195, 2001.

7. Pandian RS, Anuradha CV and Viswanathan P: Gastroprotective effect of fenugreek seeds (Trigonella foenum graecum) on experimental gastric ulcer in rats. J Ethnopharmacol 81: 393-397, 2002.

8. Bin-Hafeez B, Haque R, Parvez S, Pandey S, Sayeed I and Raisuddin S: Immunomodulatory effects of fenugreek (Trigonella foenum graecum L.) extract in mice. Int Immunopharmacol 3: 257-265, 2003.

9. Woodgate DE and Conquer JA: Effects of a stimulant-free dietary supplement on body weight and fat loss in obese adults: A six-week exploratory study. Curr Ther Res 64: 248-262, 2003. 
10. Lai F, Wen Q, Li L, Wu H and Li X: Antioxidant activities of water-soluble polysaccharide extracted from mung bean (Vigna radiata $\mathrm{L}$.) hull with ultrasonic assisted treatment. Carbohyd Polym 81: 323-329, 2010.

11. Joshi H and Kapoor VP: Cassia grandis Linn. f. seed galactomannan: Structural and crystal lographical studies. Carbohyd Res 338: 1907-1912, 2003.

12. Guo SJ, Yang YL, Zhang QM, She JB, Zhu YC and Hong JJ: Optimization of polysaccharide extraction technology from Crotalaria mucronata Desv. Seeds. Food Sci 30: 264-267, 2009.

13. Yi WS, Qin LH and Cao JB: Investigation of morphological change of green tea polysaccharides by SEM and AFM. Scanning 33: 450-454, 2011.

14. Fuentes-Perez ME, Gwynn EJ, Dillingham MS and Moreno-Herrero F: Using DNA as a fiducial marker to study SMC complex interactions with the atomic force microscope. Biophys J 102: 839-848, 2012.
15. Palacio ML, Schricker SR and Bhushan B: Block copolymer arrangement and composition effects on protein conformation using atomic force microscope-based antigen-antibody adhesion. J Biomed Mater Res A 100: 978-988, 2012.

16. Pérez S, Mazeau K and Hervé du Penhoat C: The three-dimensional structures of the pectic polysaccharides. Plant Physiol Bioch 38: 37-55, 2000.

17. Zhang M, Cui SW, Cheung PCK and Wang Q: Antitumor polysaccharides from mushrooms: A review on their isolation process, structural characteristics and antitumor activity. Trends Food Sci Tech 18: 4-19, 2007. 